\section{Estudo de base populacional da subutilização de medicamentos por motivos financeiros entre idosos na Região Metropolitana de Belo Horizonte, Minas Gerais, Brasil}

\author{
A population-based study on cost-related \\ underuse of medicines by the elderly in Greater \\ Metropolitan Belo Horizonte, Minas Gerais State, \\ Brazil
}

Tatiana Chama Borges Luz 1

Antônio Ignácio de Loyola Filho 1

Maria Fernanda Lima-Costa 1
${ }_{1}$ Núcleo de Estudos em Saúde Pública e Envelhecimento, Fundação Oswaldo Cruzl Universidade Federal de Minas Gerais, Belo Horizonte, Brasil.

\section{Correspondência}

T. C. B. Luz

Núcleo de Estudos em Saúde Pública e Envelhecimento,

Fundação Oswaldo Cruz/ Universidade Federal de Minas Gerais.

Av. Augusto de Lima 1715, Belo Horizonte, $M G$ 30190-002, Brasil.

tatianachama@cpqrr.fiocruz.br

\section{Abstract}

The aim of this study was to estimate the prevalence of cost-related underuse of medicines and associated factors in a representative sample of 1,134 elderly individuals in Greater Metropolitan Belo Horizonte, Minas Gerais State, Brazil. Prevalence of cost-related underuse was 12.9\%. After adjustments in the multivariate model, cost-related underuse was higher in individuals with income less than twice the minimum wage $(P R=0.57 ; 95 \% C I$ : 0.34-0.97), without health plan coverage $(P R=0.68$; CI95\%: 0.46-0.99), with low frequencies of physician-patient dialogue concerning health/treatment (rarely/never, $P R=$ 1.79; 95\%CI: 1.10-2.90), with fair to poor selfrated health ( $P R=1.66$; 95\%CI: $0.95-2.90$ and $P R=2.49 ; 95 \% C I: 1.38-4.48$, respectively), and with multiple comorbidities (one, $P R=2.51$; 95\%CI: 0.99-6.35; two, $P R=3.51$; 95\%CI: 1.408.72 and three or more, $P R=4.52$; 95\%CI: 1.79 11.41). Our results suggest adherence problems within this population due to a communication gap between seniors and physicians on treatment-related aspects and to the lack of health plan coverage. Especially worrisome is the high risk of cost-related underuse among elderly with poor health.

Pharmaceutical Preparations; Drug Utilization; Aged

\section{Introdução}

A subutilização é uma forma de não-adesão a um tratamento farmacoterapêutico, que ocorre quando o indivíduo demora a iniciar o tratamento prescrito, não faz uso total, ou faz uso parcial dos medicamentos, reduz a freqüência do uso ou utiliza doses menores dos fármacos indicados 1,2. Entre os idosos, a subutilização pode resultar em piora do estado de saúde, dos sintomas ${ }^{3}$ e do controle dos agravos 3,4 . Além disso, pode resultar na necessidade de prescrição adicional de doses maiores ou de terapias mais potentes que, por sua vez, podem aumentar o risco de efeitos adversos 4 , além de outros desfechos negativos como maior número de visitas a serviços de emergência, hospitalizações e morte 5 .

Apesar de não se ter, ainda, completo entendimento sobre os determinantes da subutilização dos medicamentos entre os idosos 2 , sabe-se que as barreiras ao acesso aos produtos, especialmente de ordem financeira, figuram entre os principais motivos apontados pelos usuários para a subutilização dos produtos prescritos. Estima-se que um quarto dos idosos subutilize medicamentos em virtude dos altos custos deles 6 .

A literatura tem mostrado, no entanto, que os custos dos medicamentos, por si só, não são suficientes para explicar a sua subutilização. Nesse sentido, Piette et al. 7 afirmam que essa subutilização não deve ser vista apenas como um fenômeno econômico isolado e propõem 
que a sua avaliação seja realizada em um contexto mais amplo, que leve em consideração características individuais (demográficas, sócio-econômicas e clínicas), bem como características do tratamento, do processo de cuidado e da organização do sistema de saúde no qual o indivíduo está inserido.

De fato, estudos apontam para associações entre subutilização de medicamentos por motivos financeiros e renda mais baixa $8,9,10$, ser não branco 8,10,11,12, pior condição de saúde e presença de co-morbidades 8,9,11,12. Adicionalmente, existem evidências de que a subutilização de medicamentos por motivos financeiros é menor entre idosos filiados a plano de saúde 12 , entre aqueles com menores gastos mensais com medicamentos $8,9,10$ ou que têm cobertura para obtenção dos mesmos 8,9,11,12, assim como entre aqueles que avaliam melhor a qualidade da assistência médica recebida 13,14 .

Tendo em vista que estudos epidemiológicos sobre esse tema têm permanecido restritos a países desenvolvidos e que os países menos desenvolvidos abrigam mais de $60 \%$ da população idosa mundial 15, são necessárias investigações para avaliar a extensão e os determinantes da subutilização de medicamentos por motivos financeiros nesses últimos.

O presente trabalho tem por objetivo estimar a prevalência e determinar os fatores associados à subutilização de medicamentos por motivos financeiros entre idosos residentes na Região Metropolitana de Belo Horizonte, Minas Gerais, Brasil.

\section{Metodologia}

\section{Área e população de estudo}

Este estudo é parte do inquérito de saúde da Região Metropolitana de Belo Horizonte, realizado entre maio e junho de 2003 16. A Região Metropolitana de Belo Horizonte é constituída por cerca de vinte municípios, sendo a terceira do país em tamanho populacional (4,4 milhões de habitantes) e produção econômica. O envelhecimento é uma tendência demográfica, correspondendo os idosos a $8 \%$ da população total da área estudada 16.

O inquérito de saúde foi realizado por amostragem por conglomerados em dois estágios. Os setores censitários do Instituto Brasileiro de Geografia e Estatística (IBGE) foram utilizados como unidades primárias de seleção, e o domicílio foi a unidade amostral. As perdas estimadas para o cálculo amostral foram de 20\%. Foram incluídos na amostra 7.500 domicílios, perfazendo aproxi- madamente 24 mil moradores 16. Participaram deste inquérito 5.922 domicílios (que correspondem a $79 \%$ do total) e 13.701 entrevistados (1.788 dos quais na faixa etária de 60 ou mais). A distribuição por sexo e idade dos participantes do inquérito foi semelhante à da Região Metropolitana de Belo Horizonte na faixa etária considerada. Maiores detalhes podem ser vistos em publicação anterior 16.

Foram selecionados para o presente trabalho todos os 1.386 indivíduos participantes do inquérito de saúde da Região Metropolitana de Belo Horizonte com 60 ou mais anos de idade, que necessitaram de medicamentos prescritos nos últimos 30 dias.

\section{Variável dependente}

A variável dependente do estudo foi a subutilização de medicamentos por motivos financeiros, obtida por meio da pergunta: "Nos últimos 30 dias devido a problemas financeiros, o(a) senhor(a): (1) deixou de tomar algum medicamento receitado por um médico ou dentista; (2) diminuiu o número de comprimidos que deveria tomar; (3) diminuiu a dose do remédio, partindo o comprimido ou tomando menos gotas?". Foi considerada subutilização de medicamentos por motivos financeiros a resposta afirmativa a pelo menos uma das três opções acima descritas.

\section{Variáveis independentes}

O marco teórico do presente trabalho foi o modelo comportamental de acesso de Andersen \& Newman 17. Esse modelo foi inicialmente utilizado para investigar os determinantes do uso de serviços médicos, porém, recentemente, tem sido também utilizado para investigar fatores associados à subutilização de medicamentos 18,19,20 .

Foram considerados fatores predisponentes o gênero, a idade, a cor da pele auto-referida, o estado conjugal, a escolaridade (número de anos completos), a renda pessoal mensal e o arranjo domiciliar (morar só ou não).

Os fatores capacitantes incluíram: ter uma pessoa que cuida quando está doente, freqüência com que recebe auxílio financeiro da família, freqüência com que um médico ou outro profissional de saúde esclareceu sobre a saúde ou tratamento de forma que pudesse entender, número de consultas médicas nos últimos 12 meses, ocorrência de hospitalização nos últimos 12 meses e cobertura pela medicina suplementar.

Os fatores de necessidade considerados no presente trabalho foram: auto-avaliação da saúde, número de doenças diagnosticadas por médico, capacidade funcional e número de me- 
dicamentos consumidos nos últimos 15 dias. $\mathrm{O}$ número de doenças crônicas foi baseado na referência a ter recebido diagnóstico médico para as seguintes doenças: artrite ou reumatismo, câncer, hipertensão arterial, asma ou bronquite, diabetes mellitus, angina pectoris, infarto do miocárdio, outra doença do coração, acidente vascular cerebral, doença renal crônica, doença da coluna ou das costas e depressão. A capacidade funcional foi determinada pelo relato de alguma dificuldade para realizar pelo menos uma das seguintes atividades da vida diária: caminhar de um cômodo a outro no mesmo andar, tomar banho, vestir-se, comer, ir ao banheiro e levantar-se da cama.

\section{Análise estatística}

A análise estatística foi baseada no teste do quiquadrado de Pearson e em razões de prevalência estimadas pela regressão de Poisson robusta 21. Para a análise multivariada, foram incluídas no modelo inicial todas as variáveis que, na análise univariada, apresentaram associação com a subutilização de medicamentos em nível de significância inferior a 0,20, de acordo com o teste do qui-quadrado de Pearson. A análise estatística foi realizada utilizando-se os procedimentos do programa Stata, versão 9.1 (Stata Corp., College Station, Estados Unidos) para inquéritos populacionais (procedimentos svy).

O inquérito de saúde da Região Metropolitana de Belo Horizonte foi aprovado pelo Comitê de Ética do Centro de Pesquisas René Rachou, Fundação Oswaldo Cruz (Fiocruz), Belo Horizonte, Minas Gerais, Brasil.

\section{Resultados}

Foram selecionados 1.386 participantes do inquérito de saúde da Região Metropolitana de Belo Horizonte com 60 ou mais anos de idade, que relataram ter medicamentos prescritos nos 30 dias anteriores à entrevista. Desses, 1.134 participaram do presente trabalho (252 foram excluídos por causa de dados faltantes). Os participantes e os não-participantes eram semelhantes em relação à idade [média $=70,3(\mathrm{DP}=7,9)$ e 69,9 $(\mathrm{DP}=9,6)$, respectivamente; $\mathrm{p}=0,417$ ] e ao sexo (35,6\% e 41,9\% eram homens, respectivamente; $\mathrm{p}=0,086$ ). Entre participantes, a prevalência estimada da subutilização de medicamentos por motivos financeiros foi de 12,9\%.

Na Tabela 1, estão apresentados os resultados da análise univariada da associação entre subutilização de medicamentos por motivos financeiros e características predisponentes. Associação positiva e significante foi observada para cor da pele (não branca) e associações negativas e significantes foram observadas para escolaridade (8+ anos) e renda pessoal mensal (> 2 salários mínimos)

Entre as características capacitantes, ter recebido com menor freqüência (raramente ou nunca) esclarecimentos do profissional de saúde sobre a sua saúde ou sobre o seu tratamento e ter consultado mais vezes (6 ou mais) o médico nos últimos 12 meses apresentaram-se positivamente associadas com a subutilização de medicamentos. Por outro lado, a cobertura por medicina suplementar mostrou associação negativa, sendo a subutilização $53 \%$ menor para os que são cobertos por esse benefício (Tabela 2).

Com referência às características de necessidade, pior auto-avaliação da saúde (razoável e ruim ou muito ruim), número de condições crônicas (2 e 3 ou mais) e incapacidade funcional apresentaram associações positivas e significantes com a subutilização de medicamentos (Tabela 3).

Na Tabela 4 estão apresentados os resultados finais da análise multivariada dos fatores associados à subutilização de medicamentos por motivos financeiros. As variáveis que permaneceram independente e positivamente associadas à subutilização de medicamentos foram: menor freqüência com que o médico ou outro profissional esclareceu sobre a saúde ou tratamento (raramente ou nunca), auto-avaliação da saúde como ruim ou muito ruim e número de condições crônicas (2 e 3 ou mais). Associações negativas e independentes foram observadas para renda pessoal mensal superior a dois salários mínimos e cobertura por medicina suplementar.

\section{Discussão}

A prevalência da subutilização medicamentosa por motivos financeiros encontrada na literatura é muito diversificada, abrangendo valores entre $2 \%$ e $32 \%$ 8,9,10,12,22. Na Região Metropolitana de Belo Horizonte, ela esteve dentro da variação observada nesses estudos. Ela foi semelhante aos $13 \%$ verificados por Soumerai et al. 11 junto a idosos participantes do Inquérito Medicare Current Beneficiary Survey (MCBS) e aos $13,1 \%$ apontados por Wilson et al. 19 no segundo seguimento do estudo longitudinal The Study of Choice and Quality in Senior Health Care, ambos realizados nos Estados Unidos. Diferenças de prevalência na subutilização por motivos financeiros não são fáceis de explicar: elas poderiam resultar, por exemplo, de diferenças metodológicas entre os estudos, no tocante 
Análise univariada da associação entre subutilização de medicamentos por motivos financeiros e características predisponentes. Região Metropolitana de Belo Horizonte, Minas Gerais, Brasil, 2003

\begin{tabular}{|c|c|c|c|c|c|}
\hline Características predisponentes & $\begin{array}{c}\text { Número de } \\
\text { participantes * }\end{array}$ & $\begin{array}{l}\text { Porcentagem de } \\
\text { subutilização de } \\
\text { medicamentos ** }\end{array}$ & RP & IC95\% & Valor de $p$ \\
\hline Sexo & & & & & 0,2727 \\
\hline Masculino & 49 & 11,4 & 1,00 & & \\
\hline Feminino & 101 & 13,8 & 1,22 & $0,84-1,76$ & \\
\hline Idade (anos) & & & & & 0,3266 \\
\hline $60-69$ & 88 & 14,2 & 1,00 & & \\
\hline $70-79$ & 45 & 10,5 & 0,74 & $0,51-1,08$ & \\
\hline 80 e + & 17 & 13,9 & 0,98 & $0,58-1,65$ & \\
\hline Cor da pele & & & & & 0,0411 \\
\hline Branca & 56 & 10,6 & 1,00 & & \\
\hline Não branca & 94 & 15,1 & 1,42 & $1,01-2,00$ & \\
\hline Estado conjugal & & & & & 0,5645 \\
\hline Casado & 73 & 12,5 & 1,00 & & \\
\hline Viúvo & 57 & 14,3 & 1,14 & $0,79-1,64$ & \\
\hline Solteiro/Separado & 20 & 11,0 & 0,87 & $0,53-1,46$ & \\
\hline Escolaridade (anos completos) & & & & & 0,0123 \\
\hline $0-3$ & 78 & 15,2 & 1,00 & & \\
\hline $4-7$ & 53 & 14,2 & 0,94 & $0,65-1,35$ & \\
\hline $8 \mathrm{ou}+$ & 19 & 7,2 & 0,47 & $0,28-0,79$ & \\
\hline 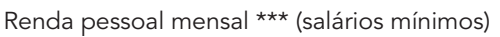 & & & & & 0,0005 \\
\hline Até 2 & 123 & 16,0 & 1,00 & & \\
\hline$>2$ & 27 & 6,7 & 0,43 & $0,27-0,69$ & \\
\hline Morar sozinho & & & & & 0,1151 \\
\hline Não & 127 & 12,2 & 1,00 & & \\
\hline Sim & 23 & 17,7 & 1,45 & $0,92-2,27$ & \\
\hline
\end{tabular}

* Número absoluto de indivíduos;

** Porcentagem ponderada pelo peso amostral;

*** Salário mínimo nacional vigente à época da pesquisa $(R \$ 240,00)$.

ao grau de detalhamento da pergunta sobre subutilização e ao período considerado na avaliação. Comparado ao nosso estudo, Wilson et al. 19, por exemplo, utilizaram uma pergunta menos detalhada ("Você alguma vez pula doses de medicamentos para que eles durem mais?") e uma janela temporal maior (um ano), e contudo, como dito acima, encontraram uma prevalência semelhante à observada entre os idosos da Região Metropolitana de Belo Horizonte. Talvez, especificidades da assistência farmacêutica prestada ao idoso no que diz respeito à qualidade da atenção farmacoterapêutica conferida no momento da prescrição 19 , assim como no que tange aos tipos de medicamentos dispensados e aos mecanismos de financiamento adotados (com ou sem contrapartida financeira do usuário) possam estar na origem das diferenças de prevalências encontradas.
Neste estudo, tanto variáveis de predisposição, de capacitação quanto de necessidade contribuíram para explicar a subutilização de medicamentos por motivos financeiros. A renda pessoal foi a única característica predisponente que se manteve independentemente associada ao evento: a subutilização de medicamentos foi menor entre idosos com renda mensal mais alta. Este resultado é consistente com o observado em populações idosas residentes em países mais desenvolvidos 8,9,10,19,20,22. No Brasil, não existem outros estudos examinando a influência da renda do idoso na subutilização de medicamentos, todavia existem fortes evidências indicativas da importância da situação financeira para o consumo de produtos farmacêuticos pelos idosos $23,24,25,26$. O gasto médio mensal com medicamentos, por exemplo, chega a comprometer um quarto da renda de metade dos idosos do país 27 . 
Análise univariada da associação entre subutilização de medicamentos por motivos financeiros e características capacitantes. Região Metropolitana de Belo Horizonte, Minas Gerais, Brasil, 2003.

\begin{tabular}{|c|c|c|c|c|c|}
\hline Características capacitantes & $\begin{array}{c}\text { Número de } \\
\text { participantes * }\end{array}$ & $\begin{array}{l}\text { Porcentagem de } \\
\text { subutilização de } \\
\text { medicamentos ** }\end{array}$ & RP & IC95\% & Valor de $\mathrm{p}$ \\
\hline Tem alguma pessoa que cuida do idoso quando ele adoece & & & & & 0,7665 \\
\hline Cônjuge & 50 & 11,5 & 1,00 & & \\
\hline Filho/Filha & 69 & 13,6 & 1,19 & $0,80-1,75$ & \\
\hline Outra pessoa & 23 & 13,2 & 1,15 & $0,69-1,90$ & \\
\hline Não tem & 6 & 16,2 & 1,41 & $0,59-3,39$ & \\
\hline Freqüência com que recebe auxílio financeiro da família & & & & & 0,4363 \\
\hline Sempre & 34 & 11,0 & 1,00 & & \\
\hline Às vezes & 41 & 14,9 & 1,35 & $0,86-2,14$ & \\
\hline Não ajuda & 74 & 12,9 & 1,17 & $0,77-1,78$ & \\
\hline \multicolumn{6}{|l|}{ Freqüência com que o médico ou outro profissional de } \\
\hline saúde, esclareceu sobre a saúde ou o tratamento & & & & & 0,0001 \\
\hline Sempre/Na maioria das vezes & 122 & 12,2 & 1,00 & & \\
\hline Raramente/Nunca & 19 & 32,7 & 2,68 & $1,72-4,19$ & \\
\hline Número de consultas médicas (últimos 12 meses) & & & & & 0,0112 \\
\hline$<3$ & 38 & 9,5 & 1,00 & & \\
\hline $3-5$ & 44 & 11,7 & 1,24 & $0,79-1,95$ & \\
\hline $6 \mathrm{ou}+$ & 65 & 17,2 & 1,81 & $1,20-2,74$ & \\
\hline Hospitalização (últimos 12 meses) & & & & & 0,5753 \\
\hline Não & 120 & 12,7 & 1,00 & & \\
\hline $\operatorname{Sim}$ & 30 & 14,2 & 1,12 & $0,75-1,68$ & \\
\hline Cobertura por medicina suplementar & & & & & 0,0001 \\
\hline Não & 109 & 17,1 & 1,00 & & \\
\hline Sim & 40 & 8,1 & 0,47 & $0,32-0,69$ & \\
\hline
\end{tabular}

* Número absoluto de indivíduos;

** Porcentagem ponderada pelo peso amostral.

Frente à pressão financeira e a falhas de distribuição de medicamentos no setor público 28 , a associação entre renda e subutilização de medicamentos é esperada.

Os indivíduos cobertos pela medicina suplementar apresentaram menor prevalência de subutilização do que aqueles que não possuem tal benefício. Pode-se supor que os idosos com um plano suplementar de saúde sejam financeiramente mais capazes de cobrir os custos dos medicamentos prescritos. Adicionalmente, esses indivíduos, por possuírem cobertura para consultas médicas e outros serviços, gastam menos com saúde, o que lhes permite custear o tratamento farmacoterapêutico ${ }^{8}$. Vale ressaltar ainda que este resultado está em concordância com outros estudos, que apontam o vínculo com planos de saúde como um importante fator explicativo do maior consumo de medicamentos $8,10,11,18,29$.
Outra associação observada em nosso estudo diz respeito à qualidade da relação médico-paciente, aqui medida pela freqüência com que o médico, ou outro profissional de saúde, esclarece o usuário sobre sua saúde e tratamento. A prevalência de subutilização por motivos financeiros foi perto de $80 \%$ maior no grupo de indivíduos que raramente ou nunca obtém tais esclarecimentos. Na literatura consultada não foram encontrados trabalhos que aferiram a relação médico-paciente de forma semelhante à nossa, o que dificulta a comparação direta dos resultados. No entanto, estudos têm demonstrado a existência de associação entre a subutilização e uma pior qualidade da relação médico-paciente, seja esta medida através de escores que englobem indicadores de comunicação, confiança, compreensão e cuidado 19 ou por meio de indicadores isolados, como a confiança 14 . Vale ressaltar que a associação observada neste trabalho está de acordo com 
Análise univariada da associação entre subutilização de medicamentos por motivos financeiros e características de necessidade. Região Metropolitana de Belo Horizonte, Minas Gerais, Brasil, 2003

\begin{tabular}{|c|c|c|c|c|c|}
\hline Características de necessidade & $\begin{array}{c}\text { Número de } \\
\text { participantes * }\end{array}$ & $\begin{array}{l}\text { Porcentagem de } \\
\text { subutilização de } \\
\text { medicamentos ** }\end{array}$ & RP & IC95\% & Valor de $\mathrm{p}$ \\
\hline Auto-avaliação de saúde & & & & & 0,0000 \\
\hline Muito boa/Boa & 26 & 6,1 & 1,00 & & \\
\hline Razoável & 70 & 13,7 & 2,23 & $1,35-3,70$ & \\
\hline Ruim/Muito ruim & 54 & 28,2 & 4,60 & $2,75-7,69$ & \\
\hline Número de condições crônicas diagnosticadas por médico & & & & & 0,0000 \\
\hline Nenhuma & 8 & 3,8 & 1,00 & & \\
\hline 1 & 30 & 8,2 & 2,16 & $0,96-4,86$ & \\
\hline 2 & 38 & 14,1 & 3,71 & $1,67-8,22$ & \\
\hline $3 \mathrm{ou}+$ & 74 & 21,2 & 5,57 & $2,60-11,91$ & \\
\hline Incapacidade funcional $\star \star \star ~$ & & & & & 0,0001 \\
\hline Não & 98 & 10,9 & 1,00 & & \\
\hline $\operatorname{Sim}$ & 52 & 21,7 & 1,99 & $1,42-2,81$ & \\
\hline Número de medicamentos usados nas últimas duas semanas & & & & & 0,1908 \\
\hline $0-1$ & 35 & 10,9 & 1,00 & & \\
\hline $2-4$ & 75 & 13,3 & 1,22 & $0,80-1,87$ & \\
\hline $5 e+$ & 37 & 17,0 & 1,56 & $0,97-2,50$ & \\
\hline
\end{tabular}

* Número absoluto de indivíduos;

** Porcentagem ponderada pelo peso amostral;

*** Atividades da vida diária incluíram caminhar de um cômodo a outro no mesmo andar, tomar banho, vestir-se, comer, ir ao banheiro e levantar da cama.

Tabela 4

Resultados estatisticamente significantes da análise multivariada dos fatores associados à subutilização de medicamentos por motivos financeiros. Região Metropolitana de Belo Horizonte, Minas Gerais, Brasil, 2003.

\begin{tabular}{|c|c|c|}
\hline Variável & RP & IC95\% \\
\hline \multicolumn{3}{|c|}{ Renda pessoal mensal (salários mínimos) * } \\
\hline Até 2 & 1,00 & \\
\hline$>2$ & 0,57 & $0,34-0,97$ \\
\hline \multicolumn{3}{|c|}{ Cobertura por medicina suplementar } \\
\hline Não & 1,00 & \\
\hline Sim & 0,68 & $0,46-0,99$ \\
\hline \multicolumn{3}{|c|}{ Freqüência com que o médico ou outro profissional de saúde, esclareceu sobre a saúde ou o tratamento } \\
\hline Sempre/Na maioria das vezes & 1,00 & \\
\hline Raramente/Nunca & 1,79 & $1,10-2,90$ \\
\hline \multicolumn{3}{|l|}{ Auto-avaliação de saúde } \\
\hline Muito boa/Boa & 1,00 & \\
\hline Razoável & 1,66 & $0,95-2,90$ \\
\hline Ruim/Muito ruim & 2,49 & $1,38-4,48$ \\
\hline \multicolumn{3}{|c|}{ Número de condições crônicas diagnosticadas por médico } \\
\hline Nenhuma & 1,00 & \\
\hline 1 & 2,51 & $0,99-6,35$ \\
\hline 2 & 3,51 & $1,40-8,72$ \\
\hline 3 ou + & 4,52 & $1,79-11,41$ \\
\hline
\end{tabular}

RP: razões de prevalência; IC95\%: intervalo de 95\% de confiança robusto, estimados pela regressão de Poisson, ajustadas pelas variáveis listadas na tabela. Categoria de referência: indivíduos que não subutilizaram medicamentos por motivos financeiros

* Salário mínimo nacional vigente à época da pesquisa ( $R \$ 240,00)$. 
trabalhos anteriores que enfatizam a importância da compreensão, pelo idoso, da sua doença e do seu tratamento, como um fator essencial para a adesão à farmacoterapia 30,31,32 .

A variação da prevalência da subutilização por motivos financeiros teve como fator explicativo mais importante a necessidade medida pela auto-avaliação do estado de saúde e número de condições crônicas. Desta forma, observa-se que ao aumento da necessidade sobrepõe-se um importante aumento da subutilização, já que tanto a piora no estado de saúde auto-referido, bem como um maior número de condições crônicas, estiveram independente e positivamente associados àquele evento. Os resultados confirmam o observado na literatura $6,8,9,10,11,12,19,22$. Idosos em piores condições de saúde tendem a receber um maior número de prescrições, com um conseqüente aumento do ônus financeiro, fato que pode explicar as associações observadas 8 .

O presente trabalho possui algumas limitações. A medida da subutilização foi obtida por auto-relato que, apesar de ter a vantagem de ser um método de fácil execução e de baixo custo, depende da memória do usuário, sendo sujeito a subestimativas de prevalência 33,34,35. Por outro lado, é importante ressaltar que a definição da subutilização de medicamentos por motivos financeiros usada neste trabalho foi semelhante à descrita em outros estudos, permitindo a comparação de resultados 9,11,36.
Outro aspecto a considerar é a impossibilidade de identificar os medicamentos subutilizados, uma vez que não foi indagado dos participantes quais foram os produtos envolvidos na subutilização. Essas informações seriam relevantes, pois as conseqüências clínicas da subutilização de medicamentos para os idosos vão depender da gravidade da(s) condição(ões) não tratada(s), da eficácia do(s) medicamento(s) subutilizado(s) e da duração da subutilização 3,37.

A presente investigação confirmou a importância dos aspectos sócio-econômicos para a subutilização medicamentosa, mas indica que sua determinação está ligada também a aspectos da qualidade na comunicação médico-paciente. $\mathrm{O}$ estudo evidencia ainda uma situação de risco, na medida em que idosos com piores condições de saúde estão subutilizando mais sua farmacoterapia.

Esses achados apontam para a necessidade de que os profissionais de saúde, ao prescreverem medicamentos, considerem a capacidade de custeio do usuário e preocupem-se em esclarecer seus pacientes sobre o tratamento e os riscos que a prática da subutilização embute, particularmente entre os idosos em piores condições de saúde. Os planejadores de saúde, por sua vez, devem desenvolver ações para reduzir os obstáculos financeiros ao uso de medicamentos, seja pelo aumento da disponibilidade dos mesmos na rede pública, seja pelo desenvolvimento de mecanismos que resultem na redução dos preços dos produtos na rede privada. 


\section{Resumo}

O objetivo deste trabalho foi estimar a prevalência e avaliar os fatores associados à subutilização de medicamentos por motivos financeiros em amostra representativa de 1.134 idosos, residentes na Região Metropolitana de Belo Horizonte, Minas Gerais, Brasil. A prevalência da subutilização foi de 12,9\%, estando independentemente associada à renda pessoal mensal inferior a dois salários mínimos $(R P=0,57$; IC95\%: 0,34-0,97), à filiação a plano privado de saúde ( $R P=$ 0,68; IC95\%: 0,46-0,99), à freqüência com que o profissional de saúde esclareceu sobre a saúdeltratamento (raramentelnunca, $R P=1,79$; IC95\%: 1,10-2,90), à auto-avaliação de saúde (razoável, $R P=1,66$; IC95\%: $0,95-2,90$ e ruim/muito ruim, $R P=2,49$; IC95\%: 1,38 $4,48)$ e ao número de condições crônicas (uma, $R P=$ 2,51; IC95\%: 0,99-6,35; duas, RP = 3,51; IC95\%: 1,408,72 e três ou mais, $R P=4,52$; IC95\%: 1,79-11,41). Os resultados confirmam a importância dos aspectos sócio-econômicos para a subutilização, mas indicam que sua determinação também está ligada à qualidade da comunicação médico-paciente. Evidencia-se ainda uma situação de risco para idosos em piores condições de saúde.

Preparações Farmacêuticas; Uso de Medicamentos; Idoso

\section{Referências}

1. Kirking DM, Lee JA, Ellis JJ, Briesacher B, McKercher PL. Patient-reported underuse of prescription medications: a comparison of nine surveys. Med Care Res Rev 2006; 63:427-46.

2. Hughes CM. Medication non-adherence in the Elderly. How big is the problem? Drugs Aging 2004; 21:793-81.

3. Heisler M, Langa KM, Eby EL, Fendrick AM, Kabeto MU, Piette JD. The health effects of restricting prescription medication use because of cost. Med Care 2004; 42:626-34.

4. Gurwitz JH, Rochon P. Considerations in designing an ideal medication-use system: lessons from caring for the elderly. Am J Health Syst Pharm 2000; 57:548-51.

5. Vik SA, Hogan DB, Patten SB, Johnson JA, Romonko-Slack L, Maxwell CJ. Medication nonadherence and subsequent risk of hospitalisation and mortality among older adults. Drugs Aging 2006; 23:345-56.

6. Safran DG, Neuman P, Schoen C, Kitchman MS, Wilson IB, Cooper B, et al. Prescription drug coverage and seniors: findings from a 2003 national survey. Health Aff 2005; 24:152-67.

\section{Colaboradores}

T. C. B. Luz foi responsável pelo delineamento do estudo, pela análise dos dados e pela redação do trabalho. A. I. Loyola Filho participou da análise dos dados, da discussão dos resultados e da revisão crítica final do artigo. M. F. Lima-Costa foi orientadora do trabalho, tendo contribuído para o delineamento do estudo e sido responsável pela supervisão da análise dos dados, da redação do trabalho e revisão crítica final do artigo.
7. Piette JD, Heisler M, Horne R, Caleb Alexander G. A conceptually based approach to understanding chronically ill patients' responses to medication cost pressures. Soc Sci Med 2006; 62:846-57.

8. Klein D, Turvey C, Wallace R. Elders who delay medication because of cost: health insurance demographic health and financial correlates. Gerontologist 2004; 44:779-87.

9. Rector TS, Venus PJ. Do drug benefits help Medicare beneficiaries afford prescribed drugs? Health Aff 2004; 23:213-22.

10. Steinman MA, Sands LP, Covinsky KE. Self-restriction of medications due to cost in seniors without prescription coverage. J Gen Intern Med 2001; 16:793-9.

11. Soumerai SB, Pierre-Jacques M, Zhang F, Ross-Degnan D, Adams AS, Gurwitz J, et al. Cost-related medication nonadherence among elderly and disabled medicare beneficiaries: a national survey 1 year before the medicare drug benefit. Arch Intern Med 2006; 166:1829-35. 
12. Taira DA, Iwane KA, Chung RS. Prescription drugs: elderly enrollee reports of financial access receipt of free samples and discussion of generic equivalents related to type of coverage. Am J Manag Care 2003; 9:305-12.

13. Wilson IB, Schoen C, Neuman P, Strollo MK, Rogers WH, Chang H, et al. Physician-patient communication about prescription medication nonadherence: a 50-state study of America's seniors. J Gen Intern Med 2007; 22:6-12.

14. Piette JD, Heisler M, Krein S, Kerr EA. The role of patient-physician trust in moderating medication nonadherence due to cost pressures. Arch Intern Med 2005; 165:1749-55.

15. United Nations. World population ageing 2007: summary tables. http://www.un.org/esa/popu lation/publications/WPA2007/SummaryTables_ new.pdf (acessado em 11/Fev/2008).

16. Lima-Costa MFF. A saúde dos adultos na Região Metropolitana de Belo Horizonte: um estudo epidemiológico de base populacional. Belo Horizonte: Núcleo de Estudos em Saúde Pública e Envelhecimento, Fundação Oswaldo Cruz/Universidade Federal de Minas Gerais; 2004

17. Andersen R, Newman JF. Societal and individual determinants of medical care utilization in the United States. Milbank Mem Fund Q Health Soc 1973; 51:95-124.

18. Kennedy J, Morgan S. A cross-national study of prescription nonadherence due to cost: data from the joint Canada-United States Survey of Health. Clin Ther 2006; 28:1217-24.

19. Wilson IB, Rogers WH, Chang H, Safran DG. Costrelated skipping of medications and other treatments among medicare beneficiaries between 1998 and 2000. J Gen Intern Med 2005; 20:715-20.

20. Saver BG, Doescher MP, Jackson JE, Fishman P. Seniors with chronic health conditions and prescription drugs: benefits wealth and health. Value Health 2004; 7:133-43.

21. Zou G. A modified poisson regression approach to prospective studies with binary data. Am J Epidemiol 2004; 159:702-6.

22. Mojtabai R, Olfson M. Medication costs adherence and health outcomes among Medicare beneficiaries. Health Aff (Millwood) 2003; 22:220-9.

23. Loyola Filho A, Uchôa E, Firmo JOA, Lima-Costa MFF. Estudo de base populacional sobre o consumo de medicamentos entre idosos: Projeto Bambuí. Cad Saúde Pública 2005; 21:545-53.
24. Carvalho MF, Pascom ARP, Souza-Júnior PRB, Damacena GN, Szwarcwald CL. Utilization of medicines by the Brazilian population, 2003. Cad Saúde Pública 2005; 21 Suppl:S100-8.

25. Lebrão ML, Laurenti R. Saúde, bem-estar e envelhecimento: o estudo SABE no Município de São Paulo. Rev Bras Epidemiol 2005; 8:127-41.

26. Coelho Filho JM, Marcopito LF, Castelo A. Perfil de utilização de medicamentos por idosos em área urbana do Nordeste do Brasil. Rev Saúde Pública 2004; 38:557-64

27. Lima-Costa MF, Barreto S, Giatt L, Uchôa E. Desigualdade social e saúde entre idosos brasileiros: um estudo baseado na Pesquisa Nacional por Amostra de Domicílios. Cad Saúde Pública 2003; 19:745-57.

28. Organização Pan-Americana da Saúde. Avaliação da assistência farmacêutica no Brasil: estrutura, processo e resultados. Brasília: Organização PanAmericana da Saúde; 2005.

29. Lima-Costa MFF, Guerra HL, Firmo JOA, Vidigal PG, Uchôa E, Barreto SM. The Bambuí Health and Aging Study (BHAS): private health plan and medical care utilization by older adults. Cad Saúde Pública 2002; 18:177-86.

30. Spiers MV, Kutzik DM, Lamar M. Variation in medication understanding among the elderly. Am J Health Syst Pharm 2004; 61:373-80.

31. Barat I, Andreasen F, Damsgaard EM. Drug therapy in the elderly: what doctors believe and patients actually do. Br J Clin Pharmacol 2001; 51:615-22.

32. Morrow D, Leirer V, Sheikh J. Adherence and medication instructions. Review and recommendations. J Am Geriatr Soc 1988; 36:1147-60.

33. Dimatteo MR. Variations in patients' adherence to medical recommendations: a quantitative review of 50 years of research. Med Care 2004; 42:200-9.

34. Vermeire E, Hearnshaw H, Van Royen P, Denekens J. Patient adherence to treatment: three decades of research. A comprehensive review. J Clin Pharm Ther 2001; 26:331-42.

35. Monane M, Monane S, Semla T. Optimal medication use in elders: key to successful aging. West J Med 1997; 167:233-5.

36. Piette JD, Heisler M, Wagner TH. Cost-related medication underuse among chronically ill adults: the treatments people forgo, how often, and who is at risk. Am J Public Health 2004; 94:1782-7.

37. Roth MT, Ivey JL. Self-reported medication use in community-residing older adults: a pilot study. Am J Geriatr Pharmacother 2005; 3:196-204.

Recebido em 14/Out/2008

Versão final reapresentada em 15/Jan/2009

Aprovado em 09/Fev/2009 